\title{
Three-body scattering in isobar ansatz
}

\section{Maxim Mai* ${ }^{*}$}

The George Washington University, Washington, DC 20052, USA

E-mail: maximmai@gwu.edu

\section{B. $\mathrm{Hu}$}

The George Washington University, Washington, DC 20052, USA

\section{Döring}

The George Washington University, Washington, DC 20052, USA

Thomas Jefferson National Accelerator Facility, Newport News, VA 23606, USA

\section{A. Pilloni}

Thomas Jefferson National Accelerator Facility, Newport News, VA 23606, USA

\section{A. Szczepaniak}

Thomas Jefferson National Accelerator Facility, Newport News, VA 23606, USA

Center for Exploration of Energy and Matter, Indiana University, Bloomington, IN 47403, USA

Physics Department, Indiana University, Bloomington, IN 47405, USA

On the basis of three-body unitarity of the S-matrix we derive a set of three-dimensional integral equations for scattering amplitude of three-to-three spinless particles. The essential input of these equations is the two-to-two scattering amplitudes, which can be parametrized conveniently by isobars in each partial wave.

XVII International Conference on Hadron Spectroscopy and Structure - Hadron2017

25-29 September, 2017

University of Salamanca, Salamanca, Spain

\footnotetext{
${ }^{*}$ Speaker.

${ }^{\dagger}$ I thank the organizers for the invitation and financial support.
} 


\section{Introduction}

Understanding of three-particle dynamics is essential for the description of new phenomena, such as light hybrids and other meson resonances with mass above $1 \mathrm{GeV}$ studied at COMPASS with pion beams [1], which also will be produced with photons at GlueX and CLAS12 [2, 3] in the near future. Similarly, one expects to reach a clearer picture of the $X Y Z$ sector [4, 5] currently explored by LHCb, BESIII, Belle and BaBar [6, 7, 8], when the three-body interactions are taken into account.

Different approaches are available to construct three-to-three amplitudes. The framework developed in $[9,10]$ is of particular interest and has inspired many three-body formalisms (see e.g., $[11,12,13,14,15])$ through the concept of hadron exchange that arises as a necessary topology in the interaction. We have taken this approach as an inspiration for our discussion of threebody scattering, see Ref. [16]. In the following we will present the general steps of the derivation of the three-to-three scattering amplitude relying on the general principles of S-matrix, such as compositeness and unitarity. At several points of this derivation we will make a comparison to the results of Refs. $[9,10]$. However, for more detailed description and comparison we refer the reader to the original publication [16].

\section{Three-body scattering amplitude}

In the following we will study the implications of the three-body unitarity and consider, therefore, for the beginning three identical, asymptotically stable, spinless, isoscalar particles of mass $M$. According to the general principle of compositeness (or cluster decomposition), the S-matrix for the transition from the incoming $\left(\left|p_{1}, p_{2}, p_{3}\right\rangle\right)$ to the outgoing three-particle states $\left(\left\langle q_{1}, q_{2}, q_{3}\right|\right)$ consists of a fully disconnected, one-time disconnected and fully connected pieces. The essential part of the second term is the yet unknown two-to-two scattering amplitude which in each partial wave can be parametrized by an isobar, see e.g. the discussion in Ref. [17]. In this context, isobar corresponds to a function $(\tau)$ of the total energy squared $(s)$ with correct analytic properties along the right-hand cut for a given set of quantum numbers (angular momentum, isospin, etc.). The correct coupling to two in/outgoing states is ensured by dissociation vertex $v$ as a cut-free function of the corresponding momenta of both asymptotic particles. In such a parametrization the one-time disconnected part of the scattering amplitude reads

$$
\left\langle q_{1}, q_{2}, q_{3}\left|\hat{T}_{d}(s)\right| p_{1}, p_{2}, p_{3}\right\rangle=\frac{1}{3 !} \sum_{n=1}^{3} \sum_{m=1}^{3} 2 E_{q_{n}}(2 \pi)^{3} \delta^{3}\left(\mathbf{q}_{n}-\mathbf{p}_{m}\right) v\left(q_{\bar{n}}, q_{\bar{n}}\right) \tau\left(\sigma\left(q_{n}\right)\right) v\left(p_{\bar{m}}, p_{\bar{m}}\right),
$$

where $P$ is the total four-momentum of the system, $\sigma(q):=(P-q)^{2}$ is the invariant mass of the isobar squared, and $\bar{n}, \overline{\bar{n}}$ are particle indices from $\{1,2,3\}$, which are neither equal among each other nor equal to $n$. In this parametrization the fully connected part can also be re-written in terms of the isobars and an unknown isobar-spectator scattering amplitude $\left\langle q_{n}|T(s)| p_{m}\right\rangle$ as

$$
\left\langle q_{1}, q_{2}, q_{3}\left|\hat{T}_{c}(s)\right| p_{1}, p_{2}, p_{3}\right\rangle=\frac{1}{3 !} \sum_{n=1}^{3} \sum_{m=1}^{3} v\left(q_{\bar{n}}, q_{\bar{n}}\right) \tau\left(\sigma\left(q_{n}\right)\right)\left\langle q_{n}|T(s)| p_{m}\right\rangle \tau\left(\sigma\left(p_{m}\right)\right) v\left(p_{\bar{m}}, p_{\bar{m}}\right) .
$$




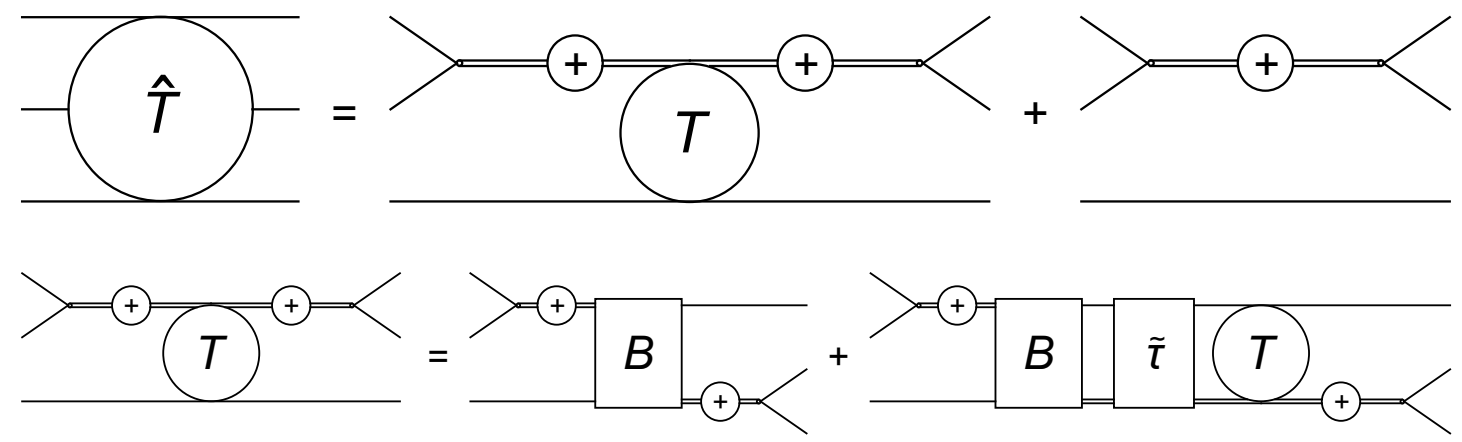

Figure 1: Top: Total scattering amplitude $\hat{T}$ consisting of a connected $\left(\hat{T}_{c}\right)$ and a disconnected contribution $\left(\hat{T}_{d}\right)$, represented by the first and second term on the right-hand side, respectively. Single lines indicate the elementary particle, double lines represent the isobar, and time runs from right to left in this and subsequent figures. The state isobar propagator $\tau$ is indicated as " + " and the (amputated) isobar-spectator scattering amplitude as $T$. Bottom: Bethe-Salpeter ansatz for the isobar-spectator amplitude $T$, where $B$ and $\tilde{\tau}$ denote the interaction kernel the isobar-spectator Green's function, respectively.

The sum of these both amplitudes yield the full three-to-three scattering amplitude

$$
\left\langle q_{1}, q_{2}, q_{3}|\hat{T}(s)| p_{1}, p_{2}, p_{3}\right\rangle=\left\langle q_{1}, q_{2}, q_{3}\left|\hat{T}_{c}(s)\right| p_{1}, p_{2}, p_{3}\right\rangle+\left\langle q_{1}, q_{2}, q_{3}\left|\hat{T}_{d}(s)\right| p_{1}, p_{2}, p_{3}\right\rangle,
$$

which is depicted pictorially in top part of Fig. 1. As indicated before the external particles are on the mass shell. Moreover, due to energy-momentum conservation, the number of free variables of a three-to-three scattering amplitude is 8 , which, e.g., in the center-of-mass frame of the three particles can be chosen to be the total energy squared (1), the angle between the incoming and outgoing spectator (1) in the scattering plane, invariant mass of the incoming and outgoing isobars (2) as well as the angle of the isobar decay plane with the scattering plane (2) and the angle between the isobar and one of its decay products (2).

In the above parametrization of the three-to-three scattering amplitude the only yet unknown, and analytically non-trivial functions are $\tau$ and $T$. They can be determined, using constraint from the S-matrix unitarity, which in terms of the T-matrix reads

$\left\langle q_{1}, q_{2}, q_{3}\left|\left(\hat{T}-\hat{T}^{\dagger}\right)\right| p_{1}, p_{2}, p_{3}\right\rangle=$

$i \int \prod_{\ell=1}^{3} \frac{\mathrm{d}^{4} k_{\ell}}{(2 \pi)^{4}}(2 \pi) \delta^{+}\left(k_{\ell}^{2}-M^{2}\right)(2 \pi)^{4} \delta^{4}\left(P-\sum_{\ell=1}^{3} k_{\ell}\right)\left\langle q_{1}, q_{2}, q_{3}\left|\hat{T}^{\dagger}\right| k_{1}, k_{2}, k_{3}\right\rangle\left\langle k_{1}, k_{2}, k_{3}|\hat{T}| p_{1}, p_{2}, p_{3}\right\rangle$,

where we have inserted a complete set of states, see the discussion of normalization of the asymptotically stable states in Ref. [16]. Furthermore, the function $\delta^{+}\left(k_{\ell}^{2}-M^{2}\right)=\delta\left(k_{\ell}^{2}-M^{2}\right) \theta\left(k_{\ell}^{0}\right)$ forces the intermediate states at momenta $k_{\ell}$ to be on-shell with positive energy.

Expanding the right-hand side of Eq. (2.4) using Eq. (2.3) we obtain four different types of diagrams, combining pairwise connected and disconnected pieces. Each one of these types breaks down again to two with a spectator carrying either the same of different particle-index connecting $T$ and $T^{\dagger}$, respectively. Therefore, eight different types of diagrams arise, see Ref. [16] for more details and a corresponding pictorial representation. The left hand side of the unitarity relation (2.4) 
can be expanded, using the Bethe-Salpeter ansatz (BSE) for the isobar-spectator interaction. The latter is depicted in the lower part of Fig. 1 and reads

$$
\left\langle q_{n}|T(s)| p_{m}\right\rangle=\left\langle q_{n}|B(s)| p_{m}\right\rangle+\int \frac{\mathrm{d}^{4} k}{(2 \pi)^{4}}\left\langle q_{n}|B(s)| k\right\rangle \tilde{\tau}(\sigma(k))\left\langle k|T(s)| p_{m}\right\rangle,
$$

where $\tilde{\tau}(\sigma(k))$ and $\left\langle q_{n}|B(s)| p_{m}\right\rangle$ denote the yet unknown isobar-spectator Green's function and the interaction kernel, respectively. In operator language this ansatz leads to exactly same eight types of diagrams as obtained on the r.h.s. of the unitarity relation (2.4). Symbolically, they read

$$
\begin{aligned}
\hat{T}-\hat{T}^{\dagger}= & v\left(\tau-\tau^{\dagger}\right) v+v\left(\tau-\tau^{\dagger}\right) T \tau v+v \tau^{\dagger} T^{\dagger}\left(\tau-\tau^{\dagger}\right) v+v \tau^{\dagger}\left(B-B^{\dagger}\right) \tau v+v \tau^{\dagger}\left(B-B^{\dagger}\right) \tilde{\tau} T \tau v \\
& +v \tau^{\dagger} T^{\dagger} \tilde{\tau}^{\dagger}\left(B-B^{\dagger}\right) \tau v+v \tau^{\dagger} T^{\dagger}\left(\tilde{\tau}-\tilde{\tau}^{\dagger}\right) T \tau v+v \tau^{\dagger} T^{\dagger} \tilde{\tau}^{\dagger}\left(B-B^{\dagger}\right) \tilde{\tau} T \tau .
\end{aligned}
$$

Therefore, the expansion of both sides of unitarity relation allows to match different types of diagrams piecewise, determining $\left(B-B^{\dagger}\right)$ and $\left(\tau-\tau^{\dagger}\right)$, when we identify $\tilde{\tau}(\sigma(k))=(2 \pi) \delta^{+}\left(k^{2}-M^{2}\right) S(\sigma(k))$, The exact matching relations are collected and presented in Ref. [16], whereas here we present only the final result of this procedure.

First, in the center of mass of the isobar $((P-k)=(\sqrt{\sigma(k)}, \mathbf{0})$ for the spectator momentum $k)$ the discontinuity of the inverse of the isobar propagator reads

$\operatorname{Disc} \frac{1}{\tau(\sigma(k))}=\frac{-i}{64 \pi^{2} K_{\mathrm{cm}}} \int \mathrm{d}^{3} \overline{\mathbf{K}} \frac{\delta\left(|\overline{\mathbf{K}}|-K_{\mathrm{cm}}\right)}{\sqrt{(\overline{\mathbf{K}})^{2}+M^{2}}}\left(v\left(\left(\begin{array}{c}\sqrt{\overline{\mathbf{K}}^{2}+M^{2}} \\ \overline{\mathbf{K}}\end{array}\right),\left(\begin{array}{c}\sqrt{\sigma(k)}-\sqrt{\overline{\mathbf{K}}^{2}+M^{2}} \\ -\overline{\mathbf{K}}\end{array}\right)\right)\right)^{2}$,

where $K_{\mathrm{cm}}=\sqrt{\sigma(k) / 4-M^{2}}$. The discontinuity of the driving term of the BSE ansatz can be rewritten in terms of the momentum transfer $Q:=(P-p-q)$ and its squared value $u:=(P-p-q)^{2}$, where $p$ and $q$ denote the incoming and outgoing spectator four-momenta, respectively,

$$
\operatorname{Disc} B(u)=2 \pi i \frac{\delta\left(E_{Q}-\sqrt{M^{2}+\mathbf{Q}^{2}}\right)}{2 \sqrt{M^{2}+\mathbf{Q}^{2}}} v(Q, q) v(Q, p) .
$$

As stated in the beginning of the paper, we assume that the dissociation vertex has no non-analyticities in the physical region. Therefore, we can utilize an un-subtracted dispersion relation to obtain

$$
\langle q|B(s)| p\rangle=\frac{v(P-p-q, q) v(P-p-q, p)}{M^{2}-u-i \varepsilon},
$$

after restoring covariance by adding a inherently real function as described in detail in Ref. [16].

In summary, after the form of the three-to-three amplitude was chosen in Eqs. (2.2) and (2.1), three-body unitarity has dictated the analytical properties of the building blocks of the BSE. Note that a different result for the driving term of the BSE was derived in Refs. [9, 10], because the imaginary part was dispersed in $s$ instead of $u$ as done here. Dispersing in $s$ implies that any other than the $s$-dependence of this term is analytical and thus does not contribute to the discontinuity. Such a treatment leads to a Blankenbecler-Sugar type of particle exchange which does not exhibit the correct analytic behavior in the $u$-channel. 


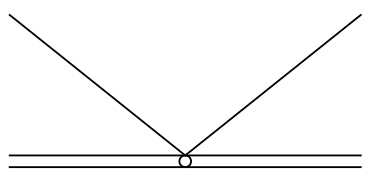

(a)

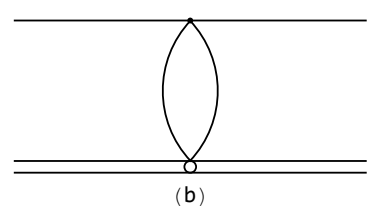

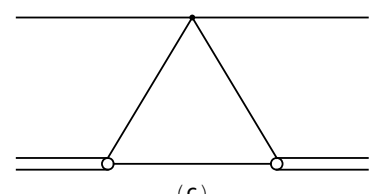

(c)

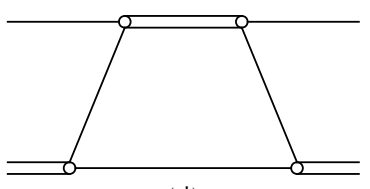

(d)

Figure 2: Additional contributions to the isobar-spectator interaction. See text for further discussion.

When we substitute the above equations into the Bethe-Salpeter Ansatz Eq. (2.5) we obtain our final expression after carrying out the integration over the zero momentum - a three-dimensional integral equation that reads

$$
\begin{aligned}
& \langle q|T(s)| p\rangle=\frac{v(P-p-q, p) v(P-p-q, q)}{M^{2}-(P-p-q)^{2}-i \varepsilon} \\
& \quad-\int \frac{\mathrm{d}^{3} \ell}{(2 \pi)^{3}} \frac{1}{2 E_{\ell}} \frac{v(P-q-\ell, \ell) v(P-q-\ell, q)}{M^{2}-(P-q-\ell)^{2}-i \varepsilon} \tau(\sigma(\ell))\langle\ell|T(s)| p\rangle,
\end{aligned}
$$

where all momenta are meant to be on-energy-shell, i.e. $E_{\ell}^{2}=\left(\ell^{2}+M^{2}\right), p_{0}^{2}=\left(\mathbf{p}^{2}+M^{2}\right)$ and $q_{0}^{2}=\left(\mathbf{q}^{2}+M^{2}\right)$. This result differs from the one of Ref. [9] by the discussed form of $B$ and by the sign of the re-scattering term which comes from a re-definition $T \rightarrow-T$ made in Ref. [9]. Note that in Eq. (2.10) $\sigma(\ell)=s+M^{2}-2 \sqrt{s} E_{\ell}$, which implies $-\infty<\sigma(\ell)<(\sqrt{s}-M)^{2}$ in the integration domain.

Finally, since three-body unitarity does only constrain the imaginary part of $B$ and $\tau$ one can add a real function without spoiling unitarity. For example, let us consider the diagrams in Fig. 2. The diagram in (a) contains a pointlike real isobar-spectator interaction and can be added to the driving term of BSE. In fact, such a contribution yield a genuine three-body force that cannot be accommodated by exchange of the light particle; through iteration in the scattering equation, such a contribution could lead to the formation of a three-body resonance. The diagram in (b) contains only $t$-channel singularities which appear as left hand cuts in the $s$-channel, and do not contribute to the unitarity equation. Conversely, the diagram in (c) actually has an imaginary part in the physical region: The three mesons in the intermediate state can go simultaneously on-shell, giving a singular contribution in $s$. Therefore, this diagram cannot be added to $B$. The top vertex describes a pointlike spectator-spectator interaction, but the only consistent treatment of the two-to-two scattering within the isobar approximation is via the exchange of an isobar. A consistent treatment of the top vertex should therefore look like the diagram in (d), which is already generated via the BSE.

\section{Discussion and Summary}

In this study, we have consequently formulated the scattering problem in terms of asymptotically stable states, including disconnected diagrams. As a result, the right-hand side of the unitarity relation acquires several new terms. These terms correspond exactly to the extra terms for the imaginary parts of the Bethe-Salpeter equation. The obtained matching relations to derive the driving term and the Green's function of the BSE are consistent and lead to a scattering equation very similar to the original results by Aaron, Amado, and Young [9, 10]. However, the scattering equation 
derived here is manifestly three-body unitary also above breakup. In the absence of three-body forces one can recast it in terms of on-shell two-to-two scattering amplitudes. In future work, the formalism will be generalized to the case of several isobars, the inclusion of 2-body channels, and (iso)spin for particles and isobars. Additionally, the three-dimensional integral form of the scattering amplitude suggests an interesting possibility to study the three-body finite volume spectrum. A first study in this direction has been already performed in Ref. [18] for the simplest case of one isobar and the most simple irreducible representation of the cubic group.

\section{References}

[1] COMPASS collaboration, P. Abbon et al., The COMPASS experiment at CERN, Nucl.Instrum.Meth. A577 (2007) 455-518, [hep-ex/ 0703049 ].

[2] GLueX collaboration, H. Al Ghoul et al., First Results from The GlueX Experiment, AIP Conf.Proc. 1735 (2016) 020001, [1512.03699].

[3] D. I. Glazier, Hadron Spectroscopy with CLAS and CLAS12, Acta Phys.Polon.Supp. 8 (2015) 503.

[4] F.-K. Guo, C. Hanhart, U.-G. Meißner, Q. Wang, Q. Zhao and B.-S. Zou, "Hadronic molecules." 2017.

[5] A. Esposito, A. Pilloni and A. D. Polosa, Multiquark Resonances, Phys. Rept. 668 (2016) 1-97, [1611.07920].

[6] LHCB collaboration, A. A. Alves, Jr. et al., The LHCb Detector at the LHC, JINST 3 (2008) S08005.

[7] BESIII collaboration, S. Fang, Hadron Spectroscopy at BESIII, Nucl.Part.Phys.Proc. 273-275 (2016) 1949-1954.

[8] Belle, BABAR collaboration, A. Bevan et al., The Physics of the B Factories, Eur.Phys.J. C74 (2014) 3026, [1406.6311].

[9] R. Aaron, R. D. Amado and J. E. Young, Relativistic three-body theory with applications to $\pi^{-} N$ scattering, Phys.Rev. 174 (1968) 2022-2032.

[10] R. Aaron, A Relativistic Three-Body Theory, pp. 139-179. Springer Berlin Heidelberg, Berlin, Heidelberg, 1977. 10.1007/978-3-642-81070-15.

[11] V. Baru, E. Epelbaum, A. A. Filin, J. Gegelia and A. V. Nefediev, Binding energy of the X(3872) at unphysical pion masses, Phys.Rev. D92 (2015) 114016, [1509.01789].

[12] M. Mai, V. Baru, E. Epelbaum and A. Rusetsky, Recoil corrections in antikaon-deuteron scattering, Phys.Rev. D91 (2015) 054016, [1411.4881].

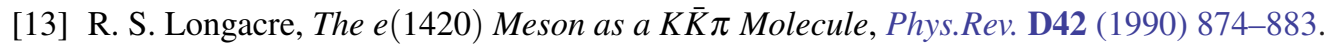

[14] S. Ceci, M. Döring, C. Hanhart, S. Krewald, U.-G. Meißner and A. Svarc, Relevance of complex branch points for partial wave analysis, Phys.Rev. C84 (2011) 015205, [1104.3490].

[15] M. Döring, C. Hanhart, F. Huang, S. Krewald and U.-G. Meißner, Analytic properties of the scattering amplitude and resonances parameters in a meson exchange model, Nucl.Phys. A829 (2009) 170-209, [0903.4337].

[16] M. Mai, B. Hu, M. Döring, A. Pilloni and A. Szczepaniak, Three-body Unitarity with Isobars Revisited, Eur. Phys. J. A53 (2017) 177, [1706.06118].

[17] P. F. Bedaque and H. W. Griesshammer, Quartet $S$ wave neutron deuteron scattering in effective field theory, Nucl. Phys. A671 (2000) 357-379, [nucl-th/9907077].

[18] M. Mai and M. Döring, Three-body Unitarity in the Finite Volume, 1709.08222. 